- GDPs suffer from work-related stress.

- A lot of the GDP work-related stress is linked to working within the NHS.

- Minor psychiatric symptoms were high, similar to doctors.

- Over half of the GDPs reported backache, headache, difficulty in sleeping and being nervy, tense or depressed.

- A third of the GDPs were overweight or obese.

- This survey highlights the need to develop interventions to reduce GDPs' work-related stress and improve their working conditions.

\title{
'It's difficult being a dentist': stress and health in the general dental practitioner
}

\author{
H. L. Myers ${ }^{1}$ and L. B. Myers ${ }^{2}$
}

\begin{abstract}
Objective The aim of the study was to investigate overall stress, workstress and health in general dental practitioners (GDPs).

Design, setting and subjects A nationwide anonymous crosssectional survey was undertaken using stratified random sampling of 2,441 GDPs in the UK.

Main outcome measures Measures included perceived stress, Work Stress Inventory for Dentists, job dissatisfaction, measures of health symptoms and health behaviour, dental and demographic information. Results The main findings were that perceived stress was significantly correlated with measures of dental stress. Work-related factors: fragility of dentist-patient relationship, time and scheduling pressures, staff and technical problems, job dissatisfaction, percentage NHS, and number of hours worked per week together explained nearly a half of GDPs overall stress in their life (linear multiple regression, adjusted $r^{2}=0.48, F(2$, $2404)=509.68, P<0.0001)$. Health behaviours such as alcohol use was associated with work stress $(r=0.18, P<0.001)$ and over a third of GDPs were overweight or obese. Sixty per cent of GDPs reported being nervy, tense or depressed, 58.3\% reported headache, $60 \%$ reported difficulty in sleeping and $48.2 \%$ reported feeling tired for no apparent reason. These were all related to work stress (one way analysis of variance, $F(1,2211)=$ $241.53 P<0.0001, F(1,2214)=86.17 P<0.0001 ; F(1,2215)=125.55$ $P<0.0001 ; F(1,2211)=209.67 P<0.0001$ respectively). Levels of minor psychiatric symptoms were high, with $32.0 \%$ of cases identified. The amount of backache was also high (reported by $68.3 \%$ of GDPs).

Conclusion A high percentage of NHS dentistry was associated with high levels of overall stress in GDPs' lives, indicating that the nature of NHS dentistry should be carefully investigated to try to improve GDPs working conditions. A comparatively large number of dentists reported
\end{abstract}

${ }^{1}$ Honourary Research Fellow, Centre for Behavioural and Social Sciences in Medicine, Royal Free and University College Medical School, University College London, Wolfson Building, 48 Riding House Street, London, W1N 8AA

${ }^{2}$ Senior Lecturer in Health Psychology, Centre for Behavioural and Social Sciences in Medicine, Royal Free and University College Medical School, University College London Wolfson Building, 48 Riding House Street, London, W1N 8AA

Correspondence to L. B. Myers

Email:I.myers@ucl.ac.uk

\section{Refereed Paper}

doi:10.1038/sj.bdj.4811476

Received 22.03.02; Accepted 12.08.03

๑) British Dental Journal 2004; 197: 89-93 high levels of psychological stress symptoms, such as being nervy, tense and depressed, showing minor psychiatric symptoms, with alcohol use being related to stress. Other factors reported which were not related to stress but may be related to the actual practice of dentistry were that a third of dentists were overweight or obese and over 60\% reported backache. Overall, these findings indicate the stressful nature of dentistry and difficulties in working conditions. The next step should be to develop interventions to help dentists to reduce stress in the dental surgery.

\section{INTRODUCTION}

Stress is a largely hidden problem within the NHS, however the 'stressors' faced by health professionals may not only affect their own mental and/or physical health but may adversely affect the quality of care that the health service offers. ${ }^{1,2}$ Being a professional within healthcare has long been identified as a high-stress occupation due to the combination of difficult working circumstances, exposure to potentially hazardous diseases, human suffering and ability to affect human life. ${ }^{3}$

Research on stress in health professions has mainly focused on doctors and nursing staff, ${ }^{4}$ with only a minority of studies studying dentists, although it is recognised that dentistry is a stressful profession. For many years, studies have suggested that dentistry generates more stress than any other profession, primarily because of the nature and working conditions of the dental surgery. ${ }^{5-7}$ Statistics on dentistry and cardiovascular disease, alcoholism, drug abuse, divorce and elevated rates of suicide suggest that the typical life of a general dental practitioner (GDP) is a stressful one. ${ }^{8-10}$ However, there has been a lack of recent comprehensive surveys investigating stress, health and the GDP. An early study carried out in the 1980s of 484 dentists in the UK by Cooper and co-workers ${ }^{6}$ identified the pattern of work stressors suffered by GDPs. However, since that time no large-scale study has been undertaken. Similarly, there has been a fairly recent study looking at health in the general dental practice, but this was a descriptive study and did not investigate stress. ${ }^{11}$

Therefore, the aim of the current study was to investigate stress and health in a large nationwide sample of GDPs from the UK. We were especially interested in finding out what factors in the dental 


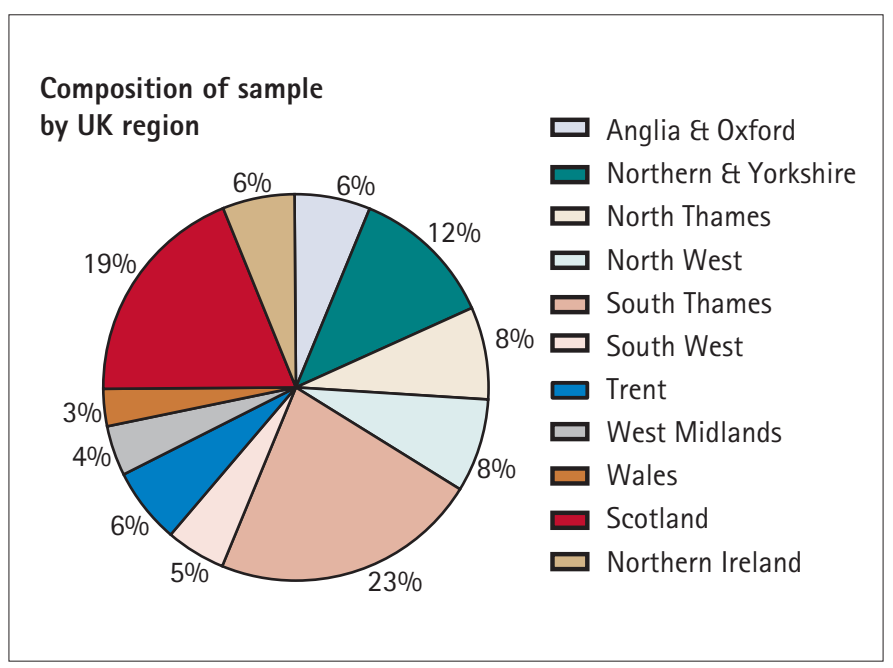

Fig. 1 Composition of sample by region

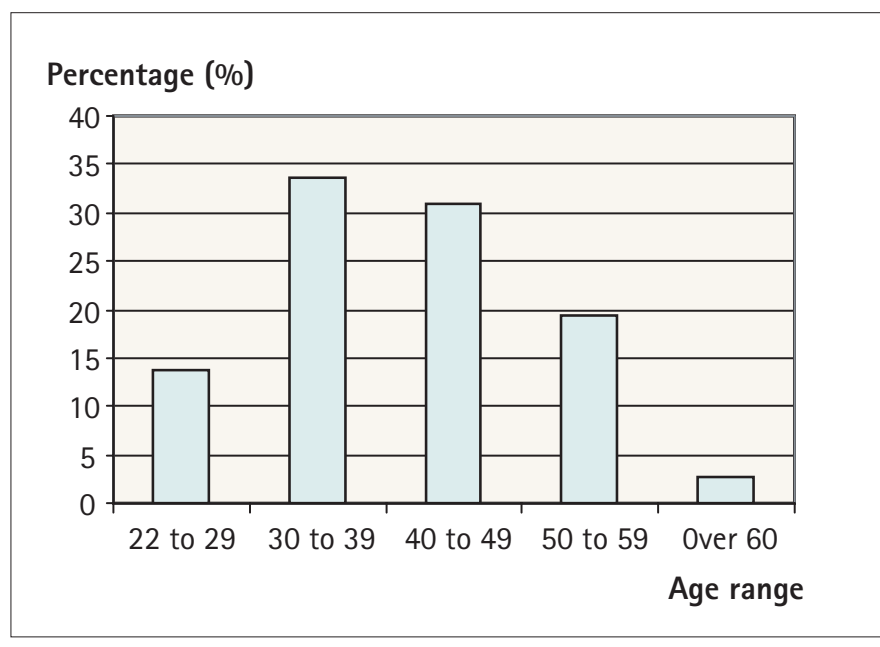

Fig. 2 Age range of GDPs

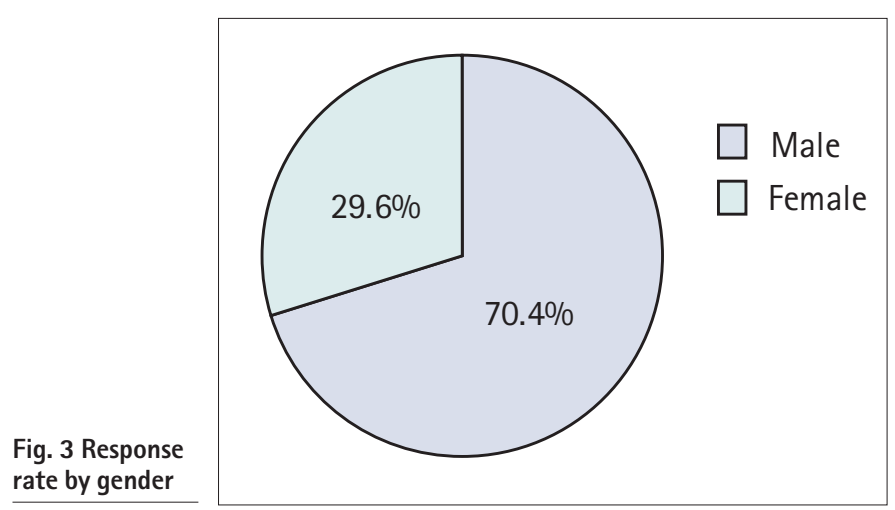

practice were related to overall stress in the GDP's life and in what way was work-related stress related to health and health behaviours.

\section{METHOD}

To achieve a representative sample from the UK, stratified random sampling was undertaken using GDPs from health authority lists. We conducted a nationwide survey of dentists from the UK during 1999/2000. A total of 4,861 questionnaires were sent out with a prepaid envelope for reply and 2,441 were returned, which gave a 50.2\% response rate. Codes were used to identify respondents to ensure anonymity and maximise the chances of candid responses. Breakdown of dentists by region is shown in Figure 1.

\section{Measures}

1. Overall stress over the last month was measured with the Perceived Stress Scale ${ }^{12}$ which is designed to measure the degree to which situations in one's life are appraised as stressful.

2. The work environment was measured with a number of questions. a) Specific dental questions (eg length of time since qualification, job description (eg principal, associate), number of dentists at the practice, percentage NHS.

b) The Work Stress Inventory for Dentists ${ }^{13}$ (WSID). This is a 30 item questionnaire describing potentially stressful work-related conditions related to dentistry. To see whether the nature of work stress in dentists had changed since the 1980s, the WSID was factor analysed (using a principal component analysis with varimax rotation, factors whose eigenvalues were one or greater were retained and loadings greater than 0.5 were considered significant. Two items were removed as they had high loading on more than one factor). Instead of five factors (or scales) in the original WSID, we found six factors, which were labelled: Factor 1 - fragility of the dentist-patient relationship, eg 'feeling underrated by patients'; Factor 2 - pay-related stressors, eg 'the piecework system of payment'; Factor 3 - time and scheduling pressures, eg 'running behind schedule'; Factor 4 - staff and technical problems, eg 'equipment breakdown and defective materials'; Factor 5 - technical problems when treating patient, eg 'actually making mistakes'; and Factor 6 - patients' difficulties going to the dentist, eg 'treating extremely nervous patients' The Cronbach's alphas for each of the new factors were all above 0.75 demonstrating a good level of internal consistency.

c) The Job Dissatisfaction Measure. ${ }^{13}$ Items included 'I would like to move to another occupation'.

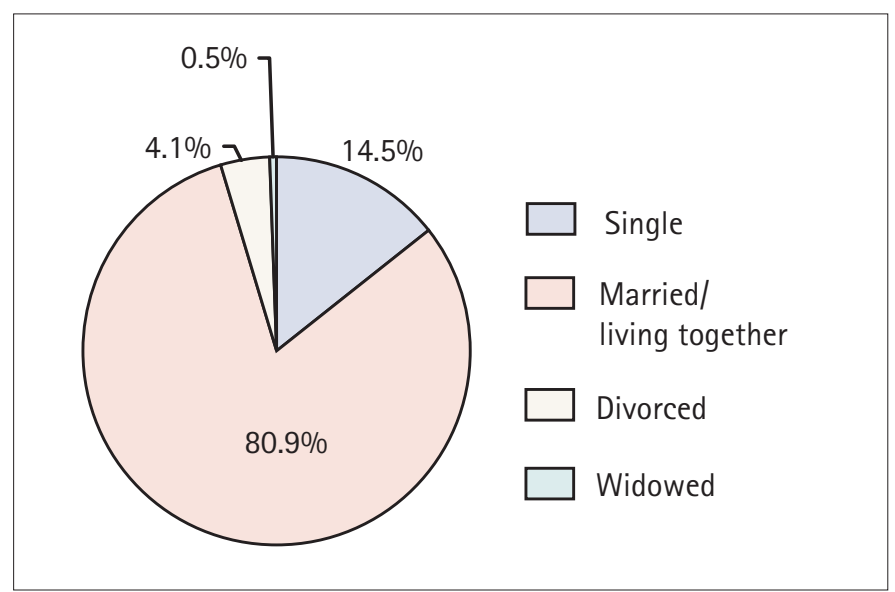

Fig. 4 Marital status of GDPs

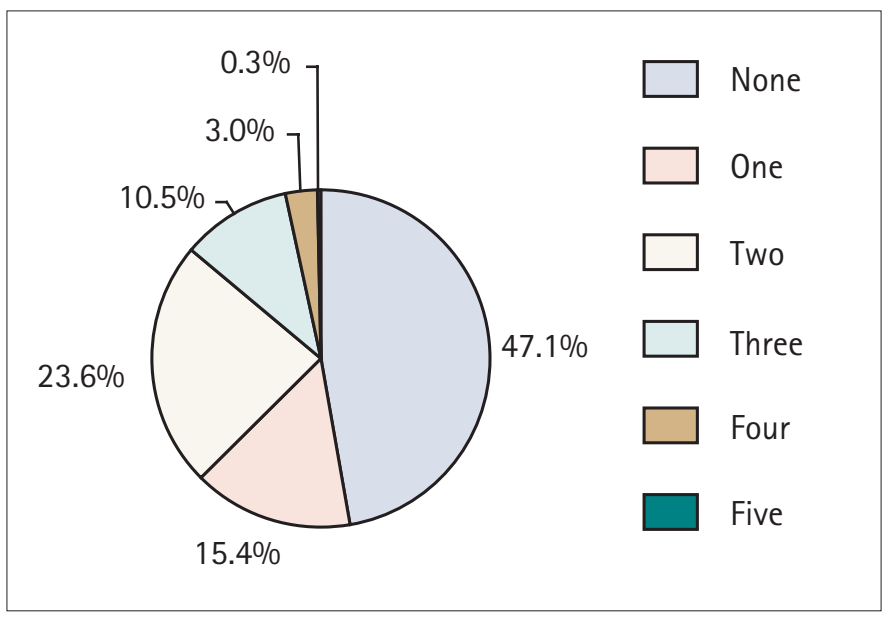

Fig. 5 Number of children dentist has (as parent) under 18 years 


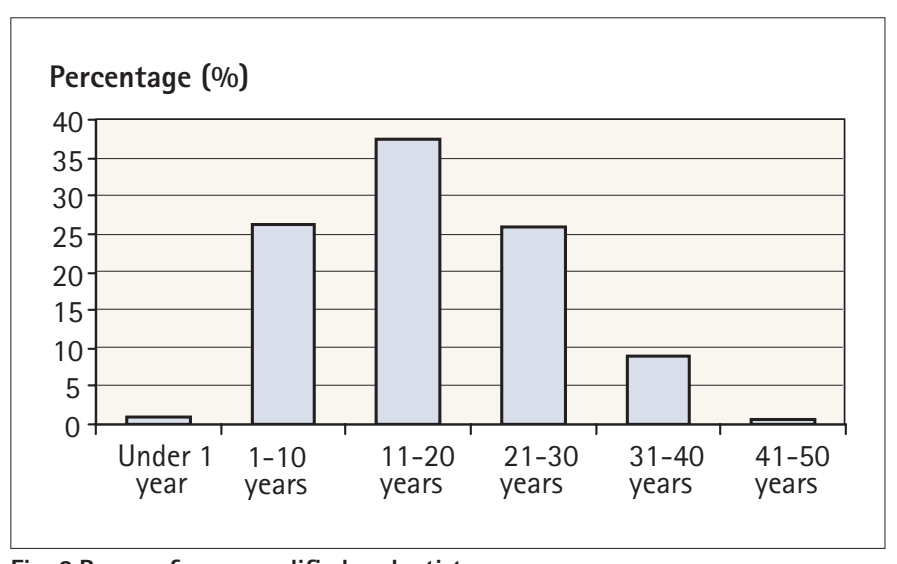

Fig. 6 Range of years qualified as dentists

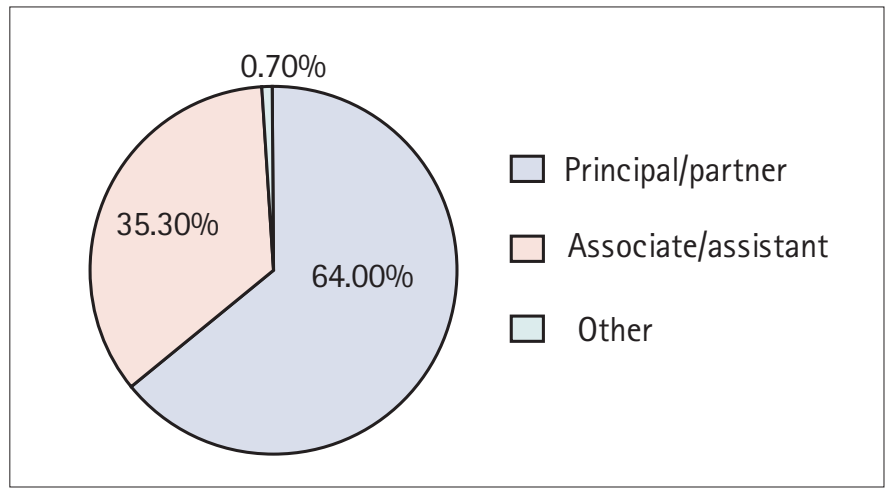

Fig. 7 Job description of GDPs

3. Demographic information. This included age, gender, marital status, number of children under 18 years.

4. Health was measured with three questionnaires:

a) General Health Questionnaire ${ }^{14}$ (GHQ-12). This is designed to detect non-psychotic psychiatric disorder and avoids physical symptoms, and was analysed to identify 'cases' of minor psychiatric illnesses.

b) Health-related behaviour. This included items on exercise, sleep, eating, alcohol, smoking, height and weight.

c) Minor ailments and symptoms. Minor ailments and symptoms over the last 14 days were measured by a checklist.

Data were analysed using SPSS. ${ }^{11}$ As well as descriptive statistics, a mixture of parametric inferential data analyses were performed including correlations and multiple regression.

\section{RESULTS}

\section{Characteristics of the individuals}

Characteristics of individuals are presented in Figures 2-9. Of the respondents, 70.4\% were male and 29.6\% female (Fig. 3). This is representative of the number of registered dentists in the UK, which is 70\% male and 30\% female.

\section{Work-stress}

Individual work stressors

We looked at the 30 individual items from the WSID to ascertain the percentage of job stressors which dentists considered the most stressful. The ten situations which dentists reported the most highly stressful are shown in Table 1.

Running behind schedule was rated as 'a lot of stress/a great deal of stress' by $68.4 \%$ of GDPs, followed by coping with difficult, uncooperative patients (64.8\%); working under constant time pressure (64.4\%); a patient having a medical emergency in the surgery $(60.8 \%)$; dissatisfied patients $(52.2 \%)$ treating extremely
Table 1 Percentage of dental stressors that GDPs rated as 'a lot of stress/a great deal of stress': the ten most stressful work events

\begin{tabular}{lc}
\hline Stressor & Percentage (\%) \\
\hline Running behind schedule & 68.40 \\
\hline Coping with difficult, uncooperative patients & 64.80 \\
\hline Working under constant time pressure & 64.40 \\
\hline A patient having a medical emergency in the surgery & 60.80 \\
\hline Dissatisfied patients & 52.20 \\
\hline Treating extremely nervous patients & 47.40 \\
\hline Seeing more patients than you want to, & 46.40 \\
\hline for income reasons & 46.20 \\
\hline Working constraints set by the NHS & 45.20 \\
\hline Working quickly to see as many patients as possible & 43.40
\end{tabular}

nervous patients $(47.4 \%)$, seeing more patients than you want to for income reasons (46.4\%); working constraints set by the NHS $(46.2 \%)$; working quickly to see as many patient as possible (45.2\%); the piecework system of payment (43.4\%).

Males compared with females worked significantly more hours per week (means, male 38.7 hours vs female 31.29 hours) and had qualified significantly earlier (means, male 18.93 years vs female 13.6 years). However, when taking these differences into account (using analysis of co-variance), there were sex differences in workrelated stress, with females compared with males reporting more lack of patient appreciation and awareness of the complex nature of the job, (means, male 2.83 vs female $3.04, \mathrm{~F}(59,2082)=3.82, P$

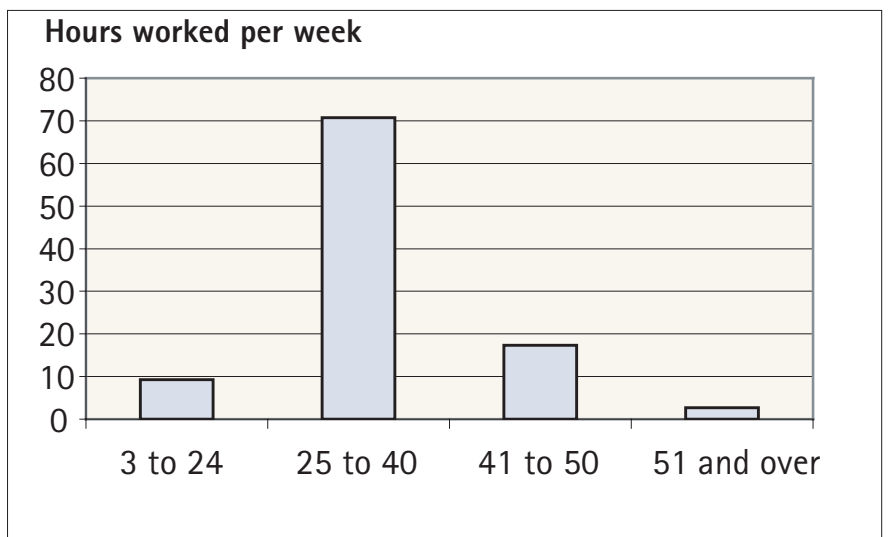

Fig. 8 Number of hours worked per week

\section{Percentage NHS}

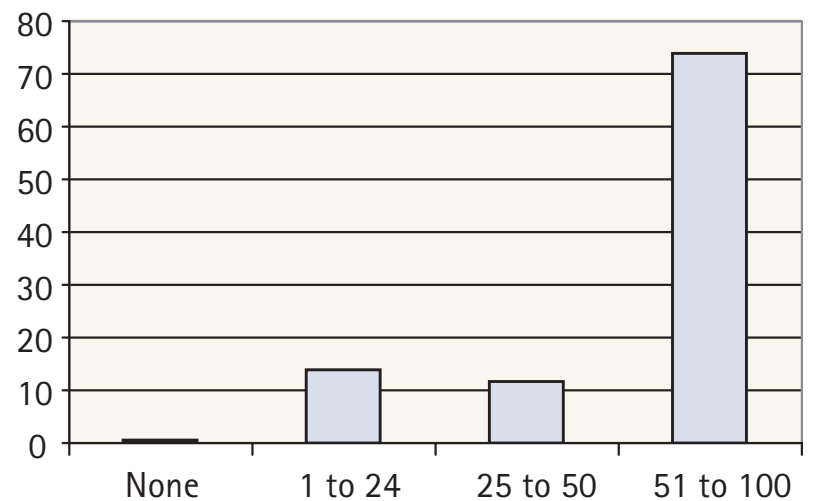

Fig. 9 Percentage NHS work undertaken by GDPs 


\section{RESEARCH}

$<0.01$ ), feeling more underrated by patients, (means, male 3.55 vs female 3.91, $F(57,2078)=4.23, P<0.01)$ and reporting more inability to meet their own expectations or standards, (means, male 2.83 vs female $3.04, \mathrm{~F}(59,2084=2.64 P<0.01)$.

There was only one significant regional difference in that the North West, Scotland, South Thames and Trent reported feeling less isolated than other regions in the UK (One Way Analysis of Variance, $\mathrm{F}(10,2398)=10.65, P<0.001)$.

For the remainder of the analyses we either looked at each of the WSID scales separately or used an overall score of work stress, that is adding all the 30 scores of the WSID together.

The relationship between work stress and demographic factors Dentists, both male and female, who were parents of children under 18 years reported significantly more overall work stress: the more children, the more work stress (one way analysis of variance, $\mathrm{F}(4,2207)=10.48, P<0.001)$.

\section{The relationship between the work environment and overall perceived stress}

Perceived stress, that is overall stress in the GDP's life, was significantly highly correlated with three of the WSID scales: Fragility of the dentist-patient relationship, $(r=0.68, P$ $<0.0001)$, time and scheduling pressures, $(0.64, P<0.0001)$ and staff and technical problems, $(r=0.54, P<0.0001)$. This means that high overall stress in a GDP's life was associated with high perceptions of: (a) a fragile dentist-patient relationship, (b) time and scheduling pressures, and (c) staff and technical problems.

The relationships with the other three WSID factors were significant but the relationship with perceived stress was less strong (pay-related stressors, $r=0.39, P<0.001$; technical problems when treating patients, $r=0.32, P<0.001$ and patients' emotional problems, $r=0.34, P<0.001$ ). This means that high overall stress in a GDP's life was associated with high perceived: (d) pay-related stressors, (e) technical problems when treating patients, and (f) patients' emotional problems.

In addition, there were high correlations with perceived stress and job dissatisfaction $(r=0.62, P<0.0001)$ percentage NHS $(r=0.56, P<0.0001)$, and hours worked per week $(r=0.42, P$ $<0.001)$. This means that high overall stress in a GDP's life was associated with (g) more job dissatisfaction, (h) a high percentage of NHS work, and (i) long hours worked per week.

The next analysis looked at whether these work-related factors could explain overall perceived stress in a GDP's life. Fragility of dentist-patient relationship, time and scheduling pressures, staff and technical problems, job dissatisfaction, percentage NHS and number of hours worked per week together explained nearly a half of GDP's overall stress in their life (Linear multiple regression, adjusted $r^{2}=0.48, \mathrm{~F}(2,2404)=509.68$, $P<0.0001)$.

\section{Health and stress}

Health behaviours

GDPs reported fairly healthy behaviours, with 59.1\% exercising at least twice a week for 30 minutes or more each time, $72.8 \%$ having breakfast daily, $60.6 \%$ sleeping on average 7 or 8 hours per night and only $19.9 \%$ snack between meals. Nine point six per cent smoke, and although over 90.4\% consume alcohol regularly, the average (mean) was low at 12 units per week only, with only $12.4 \%$ drinking over 24 units of alcohol per week. Body Mass Index indicated that $4.0 \%$ were underweight, $61 \%$ were average, but $27.6 \%$ were overweight and $7.4 \%$ were obese.

Correlations between health behaviours and overall work stress indicated that high work stress was associated with less exercise $(r=0.20, P<0.0001)$, less sleep $(r=0,19, P<0.001)$ and more alcohol use $(r=0.18, P<0.001)$.

\begin{tabular}{lcc} 
Table $\mathbf{2}$ Ten most commonly reported minor ailments in the last $\mathbf{1 4}$ days \\
\hline Symptoms & $\begin{array}{c}\text { Percentage } \\
\text { reported (\%) }\end{array}$ & $\begin{array}{c}\text { Gender } \\
\text { differences }\end{array}$ \\
\hline A backache or pains in the back & 62.3 & \\
\hline Nervy, tense or depressed & 60.0 & \\
\hline Heartburn, wind or indigestion & 59.4 & More male \\
\hline Headache* & 58.3 & More female \\
\hline Difficulty in sleeping ${ }^{*}$ & 48.6 & \\
\hline Feeling tired for no apparent reasons* & 48.2 & More female \\
\hline A cough, catarrh or phlegm & 38.4 & \\
\hline Dry, itchy or tired eyes & 34.4 & \\
\hline Blocked or runny nose & 32.9 & \\
\hline Rashes, itches or other skin trouble & 28.7 & \\
\hline *Correlated with work stress & &
\end{tabular}

General health questionnaire

We then looked at levels of minor psychiatric symptoms with the 'general health questionnaire'. Levels of minor psychiatric symptoms were high at $32.0 \%$ of cases identified.

\section{Minor health problems}

The ten most commonly reported symptoms in the last 14 days are reported in Table 2.

The main findings were as follows: backache or pains in the back was reported by $62.3 \%$ of GDPs and heartburn, wind or indigestion was reported by $60.4 \%$ of GDPs. Neither of these were related to overall work stress. Sixty per cent reported being nervy, tense or depressed, 58.3\% reported headache, $48.6 \%$ reported difficulty in sleeping, $48.2 \%$ reported feeling tired for no apparent reason and these were all related to work stress (one way analysis of variance, $\mathrm{F}(1,2211)=241.53 \quad P<0.0001, \mathrm{~F}(1,2214)=86.17$ $P<0.0001 ; \mathrm{F}(1,2215)=125.55 P<0.0001 ; \mathrm{F}(1,2211)=209.67$ $P<0.0001$, respectively).

\section{DISCUSSION}

These results highlighted the stressful nature of general practice dentistry and some potentially worrying associations with stress when working as a GDP.

Work stress was associated with overall stress in a GDP's life with work stressors contributing highly to overall stress. An important finding was that factors in the dental surgery explained nearly half of the overall stress in a GDP's life, especially fragility of dentist-patient relationship, time and scheduling pressures, staff and technical problems, job dissatisfaction, percentage NHS and number of hours worked per week. These findings highlight the stressful nature of NHS dentistry.

These findings are especially important as we found that on average GDPs spend nearly three-quarters of their time working with NHS patients. Results on the GHQ also indicated that levels of minor psychiatric symptoms were high at 32\%, similar to doctors at $27.8 \%{ }^{15}$ and higher than the general population which has been reported at $17.8 \% .{ }^{15}$ This may also be seen as an indicator of the high levels of stress in a GDP's life.

'Running behind schedule' and 'coping with difficult uncooperative patients' were the most high rated work stressors. These are obviously serious problems for dentists as they were rated by so many dentists as particularly stressful.

Over 60\% reported backache, having heartburn, wind or indigestion and these were not associated to stress, indicating general problems of working conditions in the dental surgery. Other highly reported symptoms were related to work stress feeling tense, headache, difficulty sleeping and feeling tired for no apparent reason. 
Less than 10\% of dentists smoke although the majority drink some alcohol and over a third are overweight. Although it is low, it is still surprising that almost $10.0 \%$ of dentists smoke. Over a third of dentists were either overweight or obese. This is a worry and may be related to the sedentary lifestyle of the GDP and possibly stress, as there was also an association between lack of exercise and high work stress. The use of alcohol was also related to high work stress.

The number of children under 18 years for which the GDP was a parent was related to work stress, the more children the higher the work stress. Probably, the financial pressures of children add to work pressure of dentistry. There were some sex differences in work stressors, although regional differences were minimal.

There were some limitations of this study. Firstly, the information was collected by self-report questionnaires. It has been acknowledged that some individuals are overly positive when answering such questionnaires. ${ }^{16}$ However, we tried to minimise this by making the survey totally anonymous, rather than identifying respondents (to allow for more mailings to improve response rates, which some surveys sometimes do). A further study should, in addition to self-reports, use physiological measures (eg 24 hour blood pressure monitoring) and biological measures (eg salivary cortisol) of stress. Such a study should also be longitudinal ie follow a group of dentists over time. The current study is more of a snapshot, looking at dentists at one time point, so that no conclusions can be made, for example, as to whether the link between minor health symptoms and work stress is a cause or effect: that is whether work stress is a cause of health symptoms, or health symptoms cause work stress. A longitudinal study would address this issue.

In conclusion, to the authors' knowledge, although there have been previous studies in this area ${ }^{6,9}$ this is the first compre- hensive study to investigate stress and health in a nationwide representative sample of GDPs. These findings indicate the stressful nature of dentistry, and the next step should be to develop interventions to help dentists to reduce stress in the dental surgery.

The authors would like to thank Kathleen Mulligan and to the help and support of all the health authorities who took part in this survey. The research was supported by Colgate Oral Pharmaceuticals, The Dental Directory, Rexodent, Medical Sickness Society, Dentists Provident Society and Dentists Health Support Trust.

1. British Medical Association. Stress and the medical profession. London: BMA, 1992.

2. Vincent C, Ennis M, Audley R. Medical accidents. Oxford: Oxford University Press. England, 1993.

3. Spector P. Individual differences in the job stress process of healthcare professionals. In Firth-Cozens J, Payne R, Stress in health professionals. Chichester: Wiley \& Sons, UK, 1999.

4. Firth-Cozens J, Payne R. Stress in health professionals. Chichester: Wiley \& Sons, 1999

5. Bourassa M, Baylard J F. Stress situations in Dental Practice. Can DentJ 1994; 60: 65-71.

6. Cooper C L,Watts T, Kelly K. Job satisfaction, mental health and job stressors among General Dental Practitioners in the UK. Br Dent J 1987; 24: 77-88.

7. Moore R, Brodsgaard I. Dentists' perceived stress and its relation to perceptions about the anxious patients. Community Dent Oral Epidemiol 2001: 29: 73-80.

8. Cooper C L. Dentists under pressure: a social psychological study. In Cooper C L, Marshall J (eds). White collar and professional stress. Chichester: Wiley, 1980.

9. Kent G. Stress amongst dentists. In Payne R, Firth-Cozens S. (eds). Stress and health professionals. 1st ed. London: Wiley, 1987.

10. Stack S. Occupation and suicide. Social Science Quart 2001; 82: 384-396.

11. Kay E J. Scarrott D M. A survey of dental professionals' health and well-being. $\mathrm{Br}$ Dent J 1997: 183: 340-345.

12. Cohen $S$, Kamarck T, Mermelstein R. A global measure of perceived stress. J Health Soc Behav 1983; 24: 385-396.

13. Cooper C L, Watts J, Banglioni Jr A J, Kelly M. Occupational stress amongst general practice dentists. J Occ Psychol 1988; 61: 163-174.

14. Goldberg D. General health questionnaire(GHQ-12). Windsor: NFER-NELSON, 1992.

15. Wall T, Bolden R, Carter A. Minor psychiatric disorder in NHS trust staff Occupational and gender differences. Br J Psychiat 1997; 171: 511-523.

16. Myers $L$ B. Identifying repressors: a methodological issue for health psychology. Psychol Health 2000; 15: 205-214. 